\title{
Etude de quelques cas de contaminations microbiennes importantes du lait à la ferme
}

\author{
par \\ Y.M. CHATELIN* et J. RICHARD** \\ Avec la collaboration de D. MOLLE, C. CORRE, Maryse LE SERRE, \\ Rolande DAVALLAND, P. LE TOUPIN et J. CLEMENT
}

\section{INTRODUCTION}

Le paiement du lait selon sa qualité bactériologique a été généralisé dans le but d'inciter tous les producteurs à réduire la contamination microbienne du lait livré. Pour nettoyer le matériel de traite les éleveurs ont recours à des méthodes qui ont largement fait leurs preuves, par exemple la méthode à l'eau chaude acidifiée, et qui souvent sont appliquées par l'intermédiaire de programmateurs ne laissant que peu de place à l'initiative de leurs utilisateurs. Malgré ces conditions favorables, la qualité bactériologique du lait livré aux entreprises n'est pas toujours aussi satisfaisante que ce que l'on serait en droit d'attendre.

Cela nous conduit à nous demander si la vaisselle laitière est toujours la source essentielle des micro-organismes retrouvés dans le lait ou s'il n'existe pas d'autres causes importantes d'altération de la qualité bactériologique du lait, par exemple malpropreté des mamelles, mammites, ou mauvaises conditions de conservation du lait après sa récolte.

Pour répondre à cette question une méthodologie permettant d'isoler ces causes possibles de pollution microbienne du lait a été appliquée de 1973 à 1976 dans une cinquantaine d'exploitations des environs de Rennes. L'altération de la qualité bactériologique du lait produit y était généralement la résultante de plusieurs causes ; cependant dans quelques fermes cette altération était la conséquence d'une contamination microbienne dominante. Ce sont ces cas qui ont été retenus pour la présente publication.

* ITEB, 149, rue de Bercy - 75595 Paris cedex 12 (France).

** INRA, Laboratoire de Microbiologie Laitière et de Génie Alimentaire - 78350 Jouy-en-Josas (France). 


\section{METHODE}

La méthodologie mise en œuvre reposait sur les techniques suivantes :

\section{Examen bactériologique du lait}

Pour déterminer l'influence de la propreté des mamelles, on divisait le troupeau en deux lots sensiblement égaux ; les animaux du premier lot étaient soumis à une "traite soignée " (mamelles lavées et essuyées avec soin) alors que ceux du second étaient préparés selon la technique communément pratiquée par l'éleveur. Le lait était obtenu avec du matériel de traite préalablement stérilisé à la vapeur ou nettoyé de façon efficace à l'eau chaude acidifiée [1].

Pour mettre en évidence une contamination importante du lait par des vaches atteintes de mammites on procédait à des prélèvements de lait régulièrement répartis. Les échantillons recueillis correspondaient à des fractions de 15 à 201 de lait dans le cas d'une traite en lactoduc, ou à la production individuelle des animaux lorsque l'on avait affaire à une traite en pot.

\section{Examen bactériologique des installations de traite en lactoduc}

Le pouvoir contaminant des installations de traite était estimé par un rinçage total consistant à faire circuler pendant $5 \mathrm{~min} 20$ à 301 d'eau stérile dans la machine à traire, les pulsateurs étant en fonctionnement. L'installation était mise en circuit fermé par l'intermédiaire de la canalisation de nettoyage ou, lorsque celle-ci n'existait pas, d'une canalisation stérile ajoutée par nos soins. On éliminait les sources de contamination de l'eau de rinçage extérieures au circuit à lait tels les embouts de nettoyage sales. L'eau de rinçage était préparée dans un bidon stérile et aspirée dans la canalisation en amont des postes de traite ; le bidon servait également à recueillir cette eau au sortir de la chambre de réception afin qu'elle puisse être recyclée.

Deux rinçages successifs étaient effectués afin de pouvoir distinguer une éventuelle contamination superficielle, liée par exemple à de l'eau résiduelle stagnant dans les canalisations, d'une contamination plus tenace, et donc prolongée. On a montré par ailleurs que l'eau extrait du circuit à lait pratiquement autant de micro-organismes que le lait lors de la traite [2].

\section{Numérations microbiennes}

Les échantillons recueillis étaient soumis aux dénombrements suivants : 
- flore aérobie mésophile sur milieu Plate Count Agar (Difco 479), incubation $3 \mathrm{j}$ à $30^{\circ} \mathrm{C}$;

- flore psychrotrophe sur même milieu, incubation $10 \mathrm{j}$ à $7^{\circ} \mathrm{C}$;

- bactéries coliformes sur milieu Violet Red Bile Agar (Difco 12), incubation 18 à $24 \mathrm{~h}$ à $30^{\circ} \mathrm{C}$;

- flore thermorésistante : chauffage pendant $30 \mathrm{~min}$ à $63,5^{\circ} \mathrm{C}$, $12 \mathrm{~min}$ à $75^{\circ} \mathrm{C}$ ou $10 \mathrm{~min}$ à $80^{\circ} \mathrm{C}$ du lait ou du mélange volume à volume d'eau de rinçage et de lait écrémé stérile (Skim milk Difco à 20 p. 100), puis dénombrement de la flore résistante suivant la méthode appliquée pour la flore totale. On dénombrait ainsi respectivement la flore thermorésistante «totale " constituée de toutes les bactéries mésophiles thermorésistantes (microcoques, streptocoques fécaux, Microbacterium...), la flore "fortement " thermorésistante constituée essentiellement de Microbacterium et les spores de Bacillus mésophiles.

\section{RESULTATS ET DISCUSSION}

\section{Contamination microbienne du lait par la peau des mamelles}

L'étude directe de la contamination microbienne du lait par la peau des mamelles a été réalisée dans quatre exploitations A, B, C et D. Le tableau 1 présente pour chacune de ces quatre fermes la moyenne arithmétique des numérations microbiennes faites sur les échantillons de lait obtenus d'une part avec des animaux dont les mamelles avaient été lavées très soigneusement et d'autre part avec des animaux soumis à la préparation habituelle. En outre, ce tableau indique le niveau de contamination microbienne du lait dans le tank.

On voit que les mamelles soigneusement lavées contaminent faiblement le lait : moins de 10000 germes totaux par ml, moins de 1000 micro-organismes psychrotrophes par $\mathrm{ml}$ et moins de 1000 germes thermorésistants « totaux " par ml. Les bactéries coliformes sont généralement absentes dans $1 \mathrm{ml}$.

Dans la ferme A, on n'observe pas de différence significative entre le lait des animaux traits soigneusement et celui des bêtes préparées de façon ordinaire. Cela est dû au fait que les mamelles étaient propres au moment de l'expérience.

Par contre, des mamelles sales et insuffisamment lavées apportent plusieurs dizaines de milliers de germes totaux par $\mathrm{ml}$ de lait. Nous avons même rencontré une exploitation où, à une période, les mamelles apportaient au lait plus de 200000 germes totaux par ml. Il est à noter que dans trois exploitations (B, C et D), la contamination microbienne du lait par les mamelles sales et/ou mal préparées n'affecte pas le nombre des bactéries coliformes, mais se traduit essentiellement par une augmentation significative de la flore totale, de 
TABLEAU 1

Contamination microbienne du lait par les mamelles

\begin{tabular}{|c|c|c|c|c|c|c|}
\hline \multirow{3}{*}{ Ferme } & \multirow{3}{*}{$\begin{array}{l}\text { Origine des } \\
\text { échantillons }\end{array}$} & \multicolumn{5}{|c|}{ Nombre de micro-organismes par ml } \\
\hline & & \multirow[b]{2}{*}{ Flore totale } & \multirow{2}{*}{$\begin{array}{c}\text { Flore } \\
\text { psychrotrophe }\end{array}$} & \multirow{2}{*}{$\begin{array}{l}\text { Bactéries } \\
\text { coliformes }\end{array}$} & \multicolumn{2}{|c|}{ Flore thermorésistante } \\
\hline & & & & & "Totale " & $\begin{array}{c}\text { Spores de } \\
\text { Bacillus }\end{array}$ \\
\hline A & $\begin{array}{l}\text { Traite soignée* } \\
\text { Traite ordinaire* } \\
\text { Tank }\end{array}$ & $\begin{array}{r}4800 \\
4200 \\
41000\end{array}$ & $\begin{array}{r}650 \\
2000 \\
5000\end{array}$ & $\begin{array}{r}0 \\
<\quad 10 \\
60\end{array}$ & $\begin{array}{r}190 \\
150 \\
1300\end{array}$ & $\begin{array}{l}14 \\
31 \\
42\end{array}$ \\
\hline B & $\begin{array}{l}\text { Traite soignée* } \\
\text { Traite ordinaire* } \\
\text { Tank }\end{array}$ & $\begin{array}{r}3100 \\
50000 \\
62000\end{array}$ & $\begin{array}{r}140 \\
2300 \\
4700\end{array}$ & $\begin{array}{l}<10 \\
<10 \\
70\end{array}$ & $\begin{array}{r} \\
6300 \\
4800\end{array}$ & $\begin{array}{r}11 \\
18 \\
8\end{array}$ \\
\hline $\mathrm{C}$ & $\begin{array}{l}\text { Traite soignée* } \\
\text { Traite ordinaire* } \\
\text { Tank }\end{array}$ & $\begin{array}{r}2900 \\
35000 \\
40000\end{array}$ & $\begin{array}{r}700 \\
14000 \\
12000\end{array}$ & $\begin{array}{l}<10 \\
<10 \\
<10\end{array}$ & $\begin{array}{r}<10 \\
1300 \\
850\end{array}$ & $\begin{array}{r}4 \\
17 \\
37\end{array}$ \\
\hline $\mathrm{D}$ & $\begin{array}{l}\text { Traite soignée* } \\
\text { Traite ordinaire* } \\
\text { Tank }\end{array}$ & $\begin{array}{r}9900 \\
79000 \\
90000\end{array}$ & $\begin{array}{r}300 \\
32000 \\
20000\end{array}$ & $\begin{array}{l}20 \\
40 \\
40\end{array}$ & $\begin{array}{r}300 \\
12000 \\
6800\end{array}$ & $\begin{array}{r}65 \\
300 \\
250\end{array}$ \\
\hline
\end{tabular}

* Moyenne arithmétique des résultats correspondant à des prélèvements en cours de traite. 
TABLEAU 2

Contamination microbienne du lait par les lavettes (exploitation E)

\begin{tabular}{|c|c|c|c|c|c|c|}
\hline \multirow{3}{*}{ Date } & \multirow{3}{*}{ Origine des échantillons } & \multicolumn{5}{|c|}{ Nombre de micro-organismes par ml } \\
\hline & & \multirow{2}{*}{ Flore totale } & \multirow{2}{*}{$\begin{array}{c}\text { Flore } \\
\text { psychrotrophe }\end{array}$} & \multirow{2}{*}{$\begin{array}{l}\text { Bactéries } \\
\text { coliformes }\end{array}$} & \multicolumn{2}{|c|}{ Flore thermorésistante } \\
\hline & & & & & "Totale " & $\begin{array}{l}\text { Spores de } \\
\text { Bacillus }\end{array}$ \\
\hline $21-22-8-74$ & $\begin{array}{l}\text { Traite avec matériel stérile } \\
\text { Tank }\end{array}$ & $\begin{array}{l}210000 \\
190000\end{array}$ & $\begin{array}{r}850 \\
7500\end{array}$ & $\begin{array}{l}10 \\
20\end{array}$ & $\begin{array}{l}42000 \\
28000\end{array}$ & $\begin{array}{l}30 \\
43\end{array}$ \\
\hline $28-8-74$ & $\begin{array}{l}\text { Traite avec matériel stérile. } \\
\text { Mamelles lavées soigneuse- } \\
\text { ment } \\
\text { Tank }\end{array}$ & $\begin{array}{r}8000 \\
200000\end{array}$ & $\begin{array}{r}1000 \\
80000\end{array}$ & - & $\begin{array}{r}160 \\
23000\end{array}$ & - \\
\hline $5-9-74$ & $\begin{array}{l}\text { Traite avec man.elles lavées } \\
\text { soigneusement } \\
\text { Tank }\end{array}$ & $\begin{array}{r}12000 \\
210000\end{array}$ & $\begin{array}{l}250 \\
500\end{array}$ & $\begin{array}{l}2 \\
9\end{array}$ & $\begin{array}{r}80 \\
950\end{array}$ & $\begin{array}{l}6 \\
6\end{array}$ \\
\hline $10-9-74$ & $\begin{array}{l}\text { Traite avec matériel stérile } \\
\text { Tank, traite normale, nou- } \\
\text { velles lavettes }\end{array}$ & $\begin{array}{l}6000 \\
8000\end{array}$ & $\begin{array}{r}200 \\
2200\end{array}$ & $\begin{array}{l}0 \\
1\end{array}$ & $\begin{array}{l}40 \\
20\end{array}$ & $\begin{array}{l}9 \\
9\end{array}$ \\
\hline
\end{tabular}


la flore psychrotrophe et de la flore thermorésistante « totale ». C'est ce qu'ont déjà observé Johns [3] et Labussière et al. [4].

Le niveau de contamination microbienne du lait dans le tank des exploitations B, C et D tant du point de vue de son importance que de sa nature est assez voisin de ce que l'on a trouvé pour ces mêmes exploitations dans le lait prélevé au cours de la traite des animaux préparés de façon ordinaire. On peut en déduire qu'un défaut de préparation des mamelles était dans ces fermes la cause dominante de la contamination du lait. Par contre, il n'en est pas de même dans l'exploitation $\mathrm{A}$ où le lait du tank est plus contaminé que le lait prélevé au cours de la traite.

La ferme E (tab. 2) offre un cas particulier de contamination par la peau des mamelles. La qualité bactériologique du lait produit dans cette exploitation n'était pas satisfaisante. Les résultats des numérations microbiennes faisaient apparaître des nombres élevés pour la flore totale et pour les micro-organismes thermorésistants " totaux ", mais des nombres faibles pour les bactéries coliformes. L'utilisation de matériel de traite stérile (21-22 août) ne s'est pas traduite par une réduction notable de la contamination du lait. Par contre, un lavage soigné des mamelles ( 28 août) a entraîné immédiatement une diminution importante de la flore microbienne du lait. La comparaison entre lavage soigné et lavage ordinaire des mamelles (5 septembre) prouve encore que la peau de ces mamelles était la source essentielle de contamination du lait, bien que le pis des animaux n'apparaissait pas plus sale, ni plus mal lavé que dans les exemples précédents. Cependant, dans cette dernière comparaison entre lavage ordinaire et lavage soigné des mamelles, on ne retrouve pas pour la flore thermorésistante le niveau élevé qu'elle avait auparavant. Nous avons attribué cela au changement des lavettes intervenu la semaine précédant cet examen. Les lavettes mal lavées et non désinfectées étaient auparavant conservées dans l'eau chaude durant la traite. De ce fait, elles étaient probablement le siège d'une intense prolifération microbienne et en particulier de micro-organismes thermorésistants. Depuis, nous avons eu l'occasion, dans une autre ferme, d'établir l'existence d'un tel phénomène de contamination des mamelles par les lavettes elles-mêmes.

\section{Contamination microbienne du lait par le matériel de traite}

La responsabilité prépondérante du matériel de traite dans la pollution microbienne du lait a été démontrée dans cinq exploitations (F, G, H, I et J) grâce aux rinçages totaux. Les résultats de ces examens, ainsi que ceux du lait de tank, sont rapportés dans le tableau 3.

L'exploitation $\mathrm{F}$ disposait d'une installation de traite équipée de récipients de contrôle d'un modèle ancien et, comme extracteur de lait, d'une chambre d'écluse. Cette installation était nettoyée à froid avec un iodophore [1]. Les mamelles des animaux et le matériel de 
traite étaient relativement propres. Cependant, ce dernier présentait des dépôts localisés au niveau de l'ajutage supérieur des récipients de contrôle. Ces dépôts provenaient de ce que la partie haute de ces récipients échappait partiellement aux lavages mais était malgré tout en contact avec le lait durant la traite. Par ailleurs, la chambre d'écluse n'était pas non plus parfaitement propre. Malgré cela, le lait produit dans cette ferme n'était pas très contaminé (pas plus de 20000 germes totaux par $\mathrm{ml}$ ). On notera cependant le niveau relativement élevé des bactéries coliformes et des micro-organismes psychrotrophes.

Le lait de l'exploitation $\mathrm{G}$ contenait une flore microbienne importante (380000 germes totaux par ml) essentiellement constituée de micro-organismes thermorésistants dont une proportion importante était fortement thermorésistante. Les résultats du rinçage total de la machine à traire prouvent bien que celle-ci était à l'origine de cette situation. Le démontage de l'installation a permis de révéler des dépôts importants et anciens dans les canalisations, particulièrement au niveau des coudes.

L'installation de traite de l'exploitation $\mathrm{H}$, quoique récente, était de conception très compliquée. Son nettoyage était effectué selon une méthode classique (solution chaude d'un détergent alcalin) correctement appliquée. Les données qui figurent au tableau 3 correspondent à la moyenne arithmétique des résultats de trois examens réalisés à $15 \mathrm{j}$ d'intervalle. Elles révèlent une très forte contamination du lait par des micro-organismes thermorésistants notamment du genre Microbacterium (qui ne sont pas entièrement détruits par un chauffage de $10 \mathrm{~min}$ à $80^{\circ} \mathrm{C}$ et que l'on retrouve de ce fait en partie dans le groupe correspondant normalement aux sporulés) et, dans une moindre mesure, par des bactéries coliformes. Cette contamination, comme l'attestent les rinçages totaux, mettait indiscutablement en cause la machine à traire.

Dans l'exploitation I, l'installation de traite, relativement ancienne, comportait un lactoduc en matière plastique extrêmement long dont les nombreux raccords étaient peu jointifs, ainsi qu'une chambre d'écluse comme extracteur de lait. Le matériel, nettoyé à froid avec un iodophore, paraissait propre, les mamelles des animaux étaient lavées soigneusement lors de la traite. Malgré cela, le lait de cette ferme était d'une qualité bactériologique plutôt médiocre : niveau important de la flore totale, de la flore psychrotrophe, des bactéries coliformes et des micro-organismes thermorésistants, ces derniers étant essentiellement des microcoques peu résistants au chauffage. Trois rinçages totaux réalisés à $15 \mathrm{j}$ d'intervalle ont permis de mettre en cause l'installation de traite comme facteur principal de contamination du lait. Mais il a fallu procéder à un examen complet de cette installation pour pouvoir établir la responsabilité de la chambre d'écluse et surtout des raccords du lactoduc. Ces derniers, difficiles à nettoyer correctement, étaient colonisés par des bactéries coliformes 
TABLEAU 3

Contamination microbienne du lait par les installations de traite

\begin{tabular}{|c|c|c|c|c|c|c|c|c|}
\hline \multirow{3}{*}{ Ferme } & \multirow{3}{*}{ Méthode de nettoyage } & \multirow{3}{*}{ Echantillon } & \multicolumn{6}{|c|}{ Nombre de micro-organismes par $\mathrm{ml}$} \\
\hline & & & \multirow{2}{*}{$\begin{array}{l}\text { Flore } \\
\text { totale }\end{array}$} & \multirow{2}{*}{$\begin{array}{l}\text { Flore } \\
\text { psychro- } \\
\text { trophe }\end{array}$} & \multirow{2}{*}{$\begin{array}{l}\text { Bactéries } \\
\text { coliformes }\end{array}$} & \multicolumn{3}{|c|}{ Flore thermorésistante } \\
\hline & & & & & & « Totale $»$ & $\begin{array}{c}\text { Micro- } \\
\text { bacterium }\end{array}$ & $\begin{array}{l}\text { Spores de } \\
\text { Bacillus }\end{array}$ \\
\hline $\mathrm{F}$ & A froid avec iodophore & $\begin{array}{l}\text { Rinçage total } \\
\text { Lait de tank (1 traite) }\end{array}$ & $\begin{array}{l}18000 \\
20000\end{array}$ & $\begin{array}{l}18000 \\
25000\end{array}$ & $\begin{array}{l}2000 \\
1000\end{array}$ & $\begin{array}{l}280 \\
200\end{array}$ & - & $<\begin{array}{r}10 \\
30\end{array}$ \\
\hline G & $\begin{array}{l}\text { Classique } \\
\left(60^{\circ} \mathrm{C} \text { détergent alcalin) }\right.\end{array}$ & $\begin{array}{l}\text { Rinçage total } \\
\text { Lait de tank (1 traite) }\end{array}$ & $\begin{array}{l}690000 \\
380000\end{array}$ & $\overline{3800}$ & $\begin{array}{r}250 \\
17\end{array}$ & $\begin{array}{l}430000 \\
390000\end{array}$ & $\begin{array}{l}64000 \\
50000\end{array}$ & $\begin{array}{l}160 \\
400\end{array}$ \\
\hline $\mathrm{H}$ & $\begin{array}{l}\text { Classique } \\
\left(60^{\circ} \mathrm{C} \text { détergent alcalin) }\right.\end{array}$ & $\begin{array}{l}\text { Rinçage total } \\
\text { Lait de tank ( } 2 \text { traites) }\end{array}$ & $\begin{array}{l}1700000 \\
1600000\end{array}$ & $\begin{array}{r}7800 \\
11000\end{array}$ & $\begin{array}{l}2800 \\
1200\end{array}$ & $\begin{array}{l}130000 \\
220000\end{array}$ & - & $\begin{array}{l}28000 \\
10000\end{array}$ \\
\hline I & A froid avec iodophore & $\begin{array}{l}\text { Rinçage total } \\
\text { Lait de tank ( } 2 \text { traites) }\end{array}$ & $\begin{array}{l}310000 \\
330000\end{array}$ & $\begin{array}{l}17000 \\
93000\end{array}$ & $\begin{array}{r}4700 \\
33000\end{array}$ & $\begin{array}{l}20000 \\
45000\end{array}$ & - & $\begin{array}{l}<10 \\
<10\end{array}$ \\
\hline $\mathrm{J}$ & $\begin{array}{l}\text { Classique } \\
\left(60^{\circ} \mathrm{C} \text { détergent alcalin) }\right.\end{array}$ & $\begin{array}{l}\text { Rinçage total } \\
\text { Lait de tank (1 traite) }\end{array}$ & $\begin{array}{r}82000 \\
250000\end{array}$ & $\begin{array}{r}460 \\
8000\end{array}$ & $\begin{array}{l}20000 \\
20000\end{array}$ & $\begin{array}{r}31000 \\
200000\end{array}$ & $\begin{array}{r}700 \\
70000\end{array}$ & $\begin{array}{r}500 \\
2000\end{array}$ \\
\hline
\end{tabular}


et des germes thermorésistants. Le manque de rigidité du lactoduc en matière plastique provoquait, durant la traite, le jeu de ces raccords permettant alors à une partie des micro-organismes qu'ils contenaient de passer dans le lait. Toutefois, les rinçages totaux, comme l'indique le tableau 3 , n'expliquaient pas entièrement le niveau de la contamination du lait par les germes psychrotrophes et les bactéries coliformes ; il est probable que des conditions défectueuses de conservation favorisaient une certaine multiplication de ces microorganismes.

L'installation en salle de traite de l'exploitation $\mathrm{J}$, également ancienne, paraissait propre. Elle présentait cependant un important défaut de montage (absence de piège sanitaire) de sorte que l'on avait parfois du lait dans l'intercepteur ainsi que dans la canalisation à vide. La contamination microbienne du lait de cette exploitation était importante et se traduisait par un nombre élevé de micro-organismes psychrotrophes et surtout de bactéries coliformes et de microorganismes thermorésistants. L'importance et la nature de la flore microbienne de ce lait, assez comparable à ce qui avait été observé dans les exploitations $H$ et $I$, permettaient de suspecter la machine à traire comme facteur principal de contamination du lait. Cependant, les résultats du rinçage total, sauf en ce qui concernait les bactéries coliformes, ne confirmaient que partiellement cette hypothèse. Une telle sous-estimation par le rinçage du rôle de l'installation de traite peut être due au fait que la contamination avait pour principale origine l'intercepteur et la canalisation à vide souillés par le lait et colonisés par des bactéries coliformes et des micro-organismes thermorésistants et que ces organes étaient peut-être moins aisément atteints par l'eau au cours du rinçage que par le lait durant la traite. On pouvait par ailleurs exclure une mauvaise conservation du lait puisque d'une part, on avait pris le lait d'une seule traite et d'autre part, il est bien établi que les micro-organismes thermorésistants ne se multiplient pas rapidement dans le lait cru $[5,6]$.

Dans l'exploitation $\mathrm{K}$ (tab, 4), la traite était réalisée en étable avec deux pots à terre en almasilium ; le fond de l'un d'eux présentait une corrosion profonde par piqûres. La participation de ce pottrayeur corrodé à la contamination microbienne du lait a été nettement établie par deux séries de prélèvements de lait en cours de traite effectués à près de 1 an d'intervalle. Au début, cette contamination pouvait être négligée puisque le niveau moyen de pollution du lait de cette étable était satisfaisant (moins de 50000 germes totaux par $\mathrm{ml}$ ). Par la suite, ce niveau s'est élevé à plusieurs centaines de milliers de germes totaux par $\mathrm{ml}$ (dont plus de 10000 micro-organismes thermorésistants comprenant essentiellement des microcoques). Ces résultats ont été confirmés par des frottis réalisés sur les pots-trayeurs et aussi par la stérilisation du pot corrodé qui a conduit à une réduction très nette de la contamination microbienne du lait recueilli avec ce pot. 
TABLEAU 4

Contamination microbienne du lait par un pot-trayeur (exploitation $\mathrm{K}$ )

\begin{tabular}{|c|c|c|c|c|}
\hline \multirow{4}{*}{$\begin{array}{c}\text { Echantillon } \\
\mathrm{n}^{\circ}\end{array}$} & \multicolumn{4}{|c|}{ Nombre de germes totaux par $\mathrm{ml}$} \\
\hline & \multicolumn{2}{|c|}{ Traite du 30-1-75 } & \multicolumn{2}{|c|}{ Traite du $18-12-75$} \\
\hline & \multicolumn{2}{|c|}{ Pot-trayeur } & \multicolumn{2}{|c|}{ Pot-trayeur } \\
\hline & corrodé & lisse & corrodé & lisse \\
\hline $\begin{array}{l}1 \\
2 \\
3 \\
4 \\
5 \\
6\end{array}$ & $\begin{array}{r}18000 \\
44000 \\
200000 \\
18000 \\
84000 \\
50000\end{array}$ & $\begin{array}{r}37000 \\
20000 \\
8000 \\
22000 \\
11000 \\
14000\end{array}$ & $\begin{array}{l}250000 \\
230000 \\
240000 \\
640000 \\
630000 \\
210000\end{array}$ & $\begin{array}{r}6500 \\
14000 \\
15000 \\
16000 \\
10000 \\
6500\end{array}$ \\
\hline $\begin{array}{l}\text { Moyenne des } \\
\text { résultats }\end{array}$ & 69000 & 19000 & 370000 & 11000 \\
\hline
\end{tabular}

\section{Contamination microbienne du lait par des vaches atteintes de mammites}

Il arrive que les mammites soient responsables d'une contamination importante du lait [6]. Nous avons observé ce phénomème une seule fois en 5 ans dans une des exploitations suivies.

L'exploitation L était équipée d'une installation de traite en lactoduc nettoyée par programmateur suivant une méthode classique (solution chaude d'un produit détergent alcalin). Elle produisait un lait très fortement contaminé (de 360000 à 2500000 germes totaux par $\mathrm{ml}$ suivant les périodes) (tab. 5) alors que le pouvoir contaminant de la machine à traire, déterminé par rinçage total, était faible (estimation de son apport au lait à moins de 20000 germes totaux par $\mathrm{ml}$ ). En outre, un niveau relativement bas de la flore psychrotrophe (moins de 10000 micro-organismes par ml) et des bactéries coliformes (moins de 10 par ml) permettait d'exclure un défaut de refroidissement du lait ou une défectuosité cachée du matériel. D'ailleurs, l'aspect des colonies (très petites et non pigmentées) sur milieu de dénombrement de la flore totale contrastait avec celui que l'on avait pour l'eau du rinçage total (colonies moyennes ou larges souvent pigmentées) ou 


\section{TABLEAU 5}

Contamination microbienne du lait par des vaches atteintes de mammites (exploitation L)

Prélèvements de lait au niveau du tank

\begin{tabular}{|c|c|c|c|c|c|}
\hline \multirow{3}{*}{ Date } & \multicolumn{5}{|c|}{ Nombre de micro-organismes par ml } \\
\hline & \multirow{2}{*}{ Flore totale } & \multirow{2}{*}{$\begin{array}{l}\text { Flore } \\
\text { psychro- } \\
\text { trophe }\end{array}$} & \multirow{2}{*}{$\begin{array}{l}\text { Bactéries } \\
\text { coliformes }\end{array}$} & \multicolumn{2}{|c|}{ Flore thermorésistante } \\
\hline & & & & «Totale» & $\begin{array}{c}\text { Spores de } \\
\text { Bacillus }\end{array}$ \\
\hline $13-2-75$ & 2500000 & 7200 & - & - & - \\
\hline 4-3-75 & 360000 & 4400 & $<10$ & 1300 & 60 \\
\hline 14-3-75 & 720000 & 2900 & $<10$ & 600 & 60 \\
\hline $8-4-75$ & 540000 & 1800 & $<10$ & 1800 & 190 \\
\hline
\end{tabular}

TABLEAU 6

Contamination microbienne du lait par des vaches atteintes de mammites (exploitation L)

Prélèvements de lait au cours d'une traite (par fractions de 20 l)

\begin{tabular}{|c|c|c|c|}
\hline \multirow{2}{*}{$\begin{array}{l}\text { Echantillon } \\
\mathrm{n}^{\circ}\end{array}$} & \multicolumn{3}{|c|}{ Nombre de micro-organismes par ml } \\
\hline & Flore totale & $\begin{array}{c}\text { Flore } \\
\text { psychrotrophe }\end{array}$ & $\begin{array}{l}\text { Bactéries } \\
\text { coliformes }\end{array}$ \\
\hline $\begin{array}{r}1 \\
2 \\
3 \\
4 \\
5 \\
6 \\
7 \\
8 \\
9 \\
10 \\
11 \\
12 \\
13\end{array}$ & $\begin{array}{r}21000 \\
22000 \\
11000 \\
3300 \\
15000 \\
250000 \\
70000 \\
7900 \\
11000 \\
25000 \\
240000 \\
86000 \\
62000\end{array}$ & $\begin{array}{r}1400 \\
2400 \\
900 \\
500 \\
2600 \\
2200 \\
1600 \\
1400 \\
3100 \\
8700 \\
2200 \\
3700 \\
5800\end{array}$ & $\begin{array}{r}<10 \\
20 \\
<10 \\
<10 \\
<10 \\
<10 \\
<10 \\
<10 \\
<10 \\
20 \\
<10 \\
<10 \\
<10\end{array}$ \\
\hline
\end{tabular}


celles que l'on obtient dans le cas de la multiplication à relativement basse température (colonies larges non pigmentées).

L'étude de l'évolution de la flore microbienne du lait au cours d'une traite (tab. 6) a permis d'isoler deux groupes de vaches responsables d'une élévation du niveau de pollution du lait (échantillons 5 et 11). L'examen du lait de quartiers de ces vaches a révélé que deux d'entre elles excrétaient du lait contenant plusieurs millions de microorganismes par millilitre, micro-organismes identifiés à des streptocoques du groupe pyogène. Il convient de remarquer que ces mammites n'avaient pas été décelées par l'éleveur car il n'éliminait pas les premiers jets. Le traitement à la pénicilline des quartiers infectés a entraîné une réduction notable de la contamination microbienne du lait de cette exploitation, réduction qui n'a porté que sur le niveau de la flore totale.

\section{CONCLUSIONS}

La contamination microbienne du lait à la ferme, lorsqu'elle est importante, peut avoir trois causes : les mamelles sales incorrectement lavées, le matériel de traite mal nettoyé et/ou présentant des défauts, une mauvaise conservation du lait.

Dans la plupart des exploitations étudiées l'examen visuel du matériel de traite et des pratiques de l'éleveur n'a pas permis d'établir de façon sûre l'origine principale des micro-organismes retrouvés dans le lait. En effet, hormis le cas où l'on peut déceler une faute flagrante d'hygiène, il est très difficile dans une ferme de prévoir l'influence respective des différentes erreurs observées sur la contamination microbienne du lait car deux situations apparemment semblables, rencontrées dans deux exploitations de même type, ne se traduisent pas obligatoirement par le même niveau de pollution du lait. Cela est un fait observé par un certain nombre d'auteurs $[7,8,9]$.

Pour déterminer la cause de l'altération de la qualité bactériologique du lait, il a fallu mettre en cuvre une méthodologie appropriée (prélèvements de lait au cours de la traite, examen bactériologique du matériel). Cette méthodologie relève, du fait de sa complexité, du domaine expérimental. Toutefois, les résultats qu'elle a permis d'obtenir laissent entrevoir une possibilité de révéler, à partir d'un lait de mélange, certaines causes prépondérantes de l'altération de la qualité bactériologique du lait. En effet, dans ce cas, la nature de la flore microbienne du lait paraît déterminée par l'origine de sa contamination. Comme l'ont déjà montré Thomas et al. [5], une machine à traire mal nettoyée apporte surtout des micro-organismes thermorésistants. En outre, une installation présentant des défauts entraînant une lacune dans le lavage semble se signaler par un nombre élevé de coliformes dans le lait. Une mammite, lorsqu'elle altère 
notablement la qualité bactériologique du lait, ce qui ne survient qu'avec les infections streptococciques, n'agit que sur le niveau de la flore totale. Il est bien connu, par ailleurs, qu'un défaut de refroidissement du lait ou une conservation trop longue à basse température favorisent le développement de sa flore psychrotrophe. A la lumière des résultats acquis il paraît déjà possible, en observant l'importance relative de ces groupes microbiens, d'éclairer un certain nombre de situations caractérisées par une cause dominante de contamination. Des travaux complémentaires devraient permettre de confirmer cette observation.

Bien entendu, il aurait été possible d'améliorer la qualité bactériologique du lait des exploitations étudiées sans rechercher l'origine de la contamination dominante du lait. Il aurait suffi de demander aux producteurs de respecter rigoureusement toutes les règles recommandées d'hygiène et en particulier de remédier à tous les défauts observés au niveau de la traite et du matériel de traite. C'est ce que font par exemple les Américains et les Canadiens. Cependant, cette idéalisation du système de récolte et de conservation du lait, qui implique la visite de techniciens bien informés notamment en matière de bactériologie, n'aurait pas obligatoirement été acceptée par les producteurs ; cela d'autant plus que les conseils que l'on aurait été amené à leur donner auraient été dans certains cas excessifs et que, par ailleurs, certains de leurs voisins qui " en faisaient moins " réussissaient mieux parfois. En effet, Johns [8] a montré que 30 p. 100 des exploitations livrant un lait contenant moins de 10000 germes totaux par $\mathrm{ml}$ ne suivaient pas toutes les règles d'hygiène recommandées au Canada.

Les exemples cités dans cette publication ne prétendent pas refléter la fréquence des situations de ce type en France. Ils n'ont été retenus que parce qu'ils permettaient d'analyser un certain nombre de causes possibles et importantes d'altération de la qualité bactériologique du lait. Toutefois, pour trouver ces exemples point n'a été besoin de passer en revue un nombre considérable d'exploitations. Il ne semble donc pas qu'il s'agisse de situations exceptionnelles.

\section{Ré s u mé}

On a étudié, selon une méthodologie adaptée (prélèvements de lait en cours de traite, examen bactériologique des installations de traite par rinçage total), l'origine de la contamination du lait dans cinquante exploitations. Cette publication concerne 12 fermes qui présentaient une cause importante et dominante d'altération de la qualité bactériologique du lait (peau des mamelles, lavettes, installations de traite, mammites).

Le " profil microbien » du lait semble déterminé par l'origine de la contamination de ce lait. Avec des mamelles mal lavées on observe 
une augmentation de la flore totale, des germes psychrotrophes et des micro-organismes thermorésistants, mais pas des bactéries coliformes. Une machine à traire mal nettoyée se caractérise par une flore thermorésistante élevée. Une installation de traite présentant des défauts favorise les bactéries coliformes. Les mammites streptococciques ne sont détectées que par la flore totale.

Les résultats obtenus semblent indiquer que, moyennant des améliorations, le " profil microbien » d'un lait de tank pourrait permettre de mieux déterminer l'origine de l'altération de la qualité bactériologique du lait.

\section{S u m m a r y}

SOURCES OF BACTERIAL CONTAMINATIONS OF RAW MILK

IN THE FARM

The main sources of the bacterial contamination of raw milk have been investigated using a suitable methodology (milking with sterile ustensils, bacteriological rinses of milking plants, milk sampling during milking). This paper deals with a study made in twelve farms where a large and dominant source of contamination was found, i. e. either udder skin, milking machines or cows with mastitis.

The microbial "profiles» (i. e. total, psychrotrophic, thermoduric and coliform bacterial counts in milk produced in these farms) seemed to depend on the main source of bacteria e. g. contamination by poorly washed udders was characterized by high total, but low coliform counts, milking machines having defects in design, increased the milk content of both total and coliform bacteria. On the other hand, contamination of milk by poorly cleaned milking machines led to high total and thermoduric counts.

This study shows that a bacteriological analysis of milk and the use of characteristic "profiles" may be taken as a useful aid in diagnosing the main cause of milk contamination at the farm.

Reçu pour publication en octobre 1980.

\section{Bibliographie}

[1] RichaRd (J.) (1981). - Cold cleaning with an iodophor of milk pipeline installation. Microbiological aspects. J. appl. Bact., 50 (3).

[2] RichARD (J.) (1981). - Bacteriological examination of pipeline milking machine by rinsing the entire system. J. appl. Bact. (sous presse).

[3] JoHns (C. K.) (1962). - The coliform content of raw milk as an index of udder cleanliness. XVI Int, Dairy Congr., (c), 365.

[4] Labussière (J.), Richard (J.), Combault (J. F.) et de la Chevalerie (F. A.). - (1976). - Suppression du massage et du lavage de la mamelle chez les vaches laitières. Effet sur les caractéristiques de traite et sur la qualité bactériologique du lait. Ann. Zootech., 25, 551-565. 
[5] Thomas (S. B.), Egdell (J. W.), Clegg (L. F. L.) and Cuthbert (W. A.) (1950). Thermoduric organisms in milk. Part I. A review of the litterature. Proc. Soc. appl. Bact., 13, 27-64.

[6] Cousins (C. M.) (1972). - Sources of bacteria in farm tank milk. J. Soc. Dairy Technol., 25, 200-204.

[7] Johns (C. K.), Clegg (L. F. L.), Legatt (A. G.) and Nesbitt (J. M.) (1964). - Relation between milk production conditions and results of bacteriological test with and without preliminary incubation of samples. J. Milk Food Technol., 27, 326-332.

[8] Hartley (J. C.), Reinbold (G. W.) and Vedamuthu (E. R.) (1968). - Bacterial method for evaluation of raw milk quality. A review. I. Use of pratical tests to evaluate production conditions. J. Milk Food Technol., 31, 315-323.

[9] Dabbah (R.), Moats (W. A.) and Olson (J. C.) (1971). - Evaluation of production conditions of manufacturing grade A raw milk by fieldmen ratings and by bacterial tests, J. Milk Food Technol., 34, 200-203. 